\title{
DABCO Promoted an Efficient and Convenient Synthesis of Pyrrole in Aqueous Medium
}

\author{
Harshadas Mitaram Meshram*, Vikas Madhukar Bangade, Bandi Chennakesava Reddy, \\ Gudimella Santosh Kumar, Pramod Bhagwan Thakur \\ Discovery Laboratory, Organic Chemistry Division-I, Indian Institute of Chemical Technology, Hyderabad, India \\ Email: ${ }^{*}$ hmmeshram@yahoo.com
}

Received February 11, 2012; revised March 23, 2012; accepted April 1, 2012

\begin{abstract}
The synthesis of C-substituted and $\mathrm{N}$-substituted pyrrole is described by the reaction of phenacyl bromides, pentane-2, 4-dione and amine in aqueous medium using DABCO as a catalyst. The method is very convenient and applicable for alkyl as well as aryl amines and phenacyl bromides. The procedure is amenable for the synthesis of new substituted pyrroles. Moreover, aqueous medium makes the method more eco-friendly.
\end{abstract}

Keywords: Phenacyl Bromide; Pentane-2,4-Dione; Amines; DABCO; Water; Pyrroles

\section{Introduction}

The pyrrole ring is found in porphyrin, bile pigment and in bioactive natural products [1-3]. Pyrrole oligomers also have applications as conducting material $[4,5]$. Due to their diverse potential applications in biological and pharmaceutical activities [6-9], there is a continuous interest for the synthesis of pyrroles by simple methods, though there are numerous methods $[10,11]$ known. Three components coupling of amines, aldehydes and nitroalkanes catalyzed by $\mathrm{SmI}_{2}$ is reported lead to pyrroles in fair yield [12]. Recently, a synthesis of pyrroles was accomplished by the multicomponent reaction of phenacyl bromide, amine and pentane-2,4-dione in presence of $\beta$ cyclodextrin in $\mathrm{H}_{2} \mathrm{O}$ [13]. However, the reported method has limitations to phenacyl bromide and aryl amines. In addition, some of the procedures need longer reaction times, catalyst and higher boiling solvents or chlorinated solvent which eventually result in the generation of substantial amount of wastes. In view of this, there is need to explore the systematic study of this approach to evolve a general method using environmentally benign solvent.

Recently, more emphasis is given to formulate the reactions based on green chemistry principles [14]. Particularly, to avoid the use of toxic and chlorinated hydrocarbon solvents because of direct concern with environmental hazardous. The use of aqueous medium is an intriguing step in organic reactions which was attracted the increasing interest because of environmental and economical issue [15]. In this context there is significant research awareness to revisit the organic reactions in aqueous me-

\footnotetext{
${ }^{*}$ Corresponding author.
}

dium because it is safest and abundantly available solvent. Very recently, 1,4-diaza-bicyclo[2.2.2] octane (DABCO) has emerged as a promoter for various organic reactions [16-21]. It is an organic base acts as nucleophile, exceedingly soluble in water.

In continuation of our research [22-28] program to develop environmentally friendly reactions, herein we wish to report a simple, practical and general three component reaction for the construction of pyrrole derivatives by the reaction of substituted phenacyl bromide, aryl/alkyl amines with pentane-2,4-dione in aqueous medium in the presence of DABCO (Scheme 1).

\section{Results and Discussions}

The initial reaction of phenacyl bromide $(1 \mathrm{mmol})$, phenyl ethylamine $(1 \mathrm{mmol})$ and pentane-2,4-dione $(1 \mathrm{mmol})$ in water $(5 \mathrm{ml})$ at room temperature gave low yield $(20 \%)$ of corresponding pyrrole even after stirring for extended time $(10 \mathrm{hr})$. Even after heating the reaction mixture at $60^{\circ} \mathrm{C}$ for $5 \mathrm{hr}$ did not improve the yield. However, the reaction was forced to completion by the addition of catalytic amount of DABCO (10 mol\%) and desired pyrrole was isolated in high yield (84\%).

After extensive screening of the mole ratio $(5,10,15$ mol\%) of DABCO, we found that $10 \mathrm{~mol} \%$ was suitable for maximum conversion of product. The increase in the mole ratio of DABCO did not improve the yield. Among the solvents like, water, THF, DCM, methanol, water appears to give the best result. This remarkable improvement by the catalytic activity of DABCO provided an incentive for further study of reactions with other amines. 


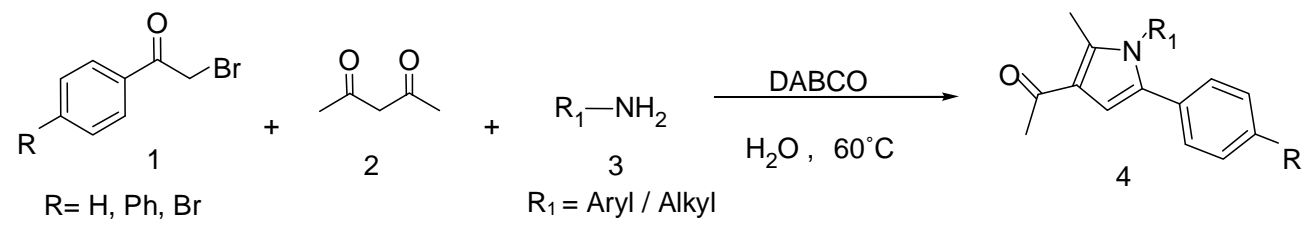

Scheme 1. Synthesis of pyrrole.

We have studied the reaction of various substituted anilines and phenacyl bromides to demonstrate the generality of method and the results are summarized in Figure 1. Aromatic amine bearing electron donating substituents like, methoxy (entries 9 and 10), hydroxy (entries 7, 8 and 25), smoothly reacted with phenacyl bromides to furnish desired product in high yield. Similarly, halogen substituted anilines (entries 11, 12, 15, 16, 18, 19, 20, 23 and 24) reacted analogously with phenacyl bromides and resulted into expected pyrroles in good yield. In addition to this, the reaction of benzylic amines with phenacyl halides underwent smoothly to give pyrroles under optimized conditions [26]. For example, benzyl amine (entry 13) and phenyl ethylamine (entries 5 and 22) also reacted analogously under standard condition to afford expected product in high yield. Next we examined the aliphatic amine having different length of alkyl chains. The reaction of methylamine (entries 2 and 21) gave methyl substituted pyrroles in good yield. However, alkyl amines with chain length $\mathrm{C}_{4}$ to $\mathrm{C}_{8}$ (entries 3 and 4 ) gave moderate yield of pyrrole. It was noted that the order of reactivity of aliphatic amine decreases as the chain length increases. Further, the application of this protocol was extended to substituted phenacyl bromides. 4-Bromophenacyl bromide (entries 20 - 25) and 4-phenyl substituted phenacyl bromide (entry 19) reacted with anilines afforded high yield of expected product. The present method provided useful alternative over earlier slurry methods in terms of reaction time and yield for the same transformation. In addition to this, the present method avoids the use of metallic base or expensive reagents. The method is clean and products were obtained in high yield. It is also worthy to mention that the protocol provides access for the synthesis of new functional pyrroles which are not prepared earlier (entries 2 - 5, 7, 8, 11, 15, 16, 18 - 25) (Figure 1).

Possible mechanism for the formation of pyrrole is shown in the Scheme 2. We presume that initially pentane-2,4-dione 2 reacts with amine 3 to form the unsaturated amino ketone 5 and tautomerised to form intermediate $6[29,30]$. DABCO reacts with phenacyl bromide 1 and forms quaternary salt 7 [31], which subsequently react with intermediate 6 and form intermediate 8 . Further the internal cyclization and dehydration of intermediate 8 resulted into expected product 4 (Scheme 2).

\section{Experimental}

\subsection{Instruments and Characterization}

All reactions were carried out without any special precautions in an atmosphere of air. Chemicals were purchased from Fluka and S. D. Fine Chemicals. TLC: precoated silica gel plates $\left(60 F_{254}, 0.2 \mathrm{~mm}\right.$ layer; E. Merk ${ }^{1} \mathrm{H}-\mathrm{NMR}$ Spectra: Varian 200 or Bruker 300 spectrometer; in $\mathrm{CDCl}_{3} ; \delta$ in ppm, $\mathrm{J}$ in Hz. Mass spectra: VG Autospec; in $m / z$.

\subsection{General Procedure}

A mixture of phenacyl bromide (1 mmol), acetyl acetone (1 mmol), amine (1 mmol) and DABCO (5 mol\%) was stirred in $5 \mathrm{ml}$ water at $60^{\circ} \mathrm{C}$ for the stipulated time (Figure 1). After completion of the reaction, as indicated by TLC, the reaction mixture was diluted with water and extracted with ethyl acetate $(2 \times 10 \mathrm{ml})$. The combined organic layers were washed with brine solution and dried over $\mathrm{Na}_{2} \mathrm{SO}_{4}$. The organic layer was concentrated under vacuum and the resulting product was directly charged on a silica gel (Merck, 60 - 120 mesh) column and eluted with a mixture of ethyl acetate/n-hexane (1:9) to afford the corresponding pure product. All the products were characterized by IR, mass and NMR spectroscopy.

\subsection{Spectral Data of New Compounds Is Given Below}

1-(1,2-dimethyl-5-phenyl-1H-pyrrol-3-yl)ethanone (4b): Yellow oil; ${ }^{1} \mathrm{H}$ NMR (300 MHz, $\mathrm{CDCl}_{3}$ ): $\delta$ (ppm) $7.40-$ $7.21(\mathrm{~m}, 5 \mathrm{H}), 6.39(\mathrm{~s}, 1 \mathrm{H}), 3.56(\mathrm{~s}, 3 \mathrm{H}), 2.47(\mathrm{~s}, 3 \mathrm{H})$, 1.94 (s, 3H); MS (ESI): $\mathrm{m} / \mathrm{z}=214[\mathrm{M}+1]^{+}$; IR (KBr): 2924, 1654, 1599, 1405, $1223 \mathrm{~cm}^{-1}$.

1-(1-butyl-2-methyl-5-phenyl-1H-pyrrol-3-yl)ethan one (4c): Yellow oil; ${ }^{1} \mathrm{H}$ NMR $\left(300 \mathrm{MHz}, \mathrm{CDCl}_{3}\right): \delta$ (ppm) $7.41-7.11(\mathrm{~m}, 5 \mathrm{H}), 6.42(\mathrm{~s}, 1 \mathrm{H}), 3.91(\mathrm{t}, \mathrm{J}=7 \mathrm{~Hz}$, 2H), $2.47(\mathrm{~s}, 3 \mathrm{H}), 2.37(\mathrm{~s}, 3 \mathrm{H}), 1.72(\mathrm{~m}, 2 \mathrm{H}), 1.02-0.95$ (m, 2H), $0.80(\mathrm{t}, \mathrm{J}=7 \mathrm{~Hz}, 3 \mathrm{H}) ; \mathrm{MS}(\mathrm{ESI}): \mathrm{m} / \mathrm{z}=256[\mathrm{M}$ $+1]^{+}$; IR (KBr): 2940, 1665, 1605, 1490, $755 \mathrm{~cm}^{-1}$.

1-(2-methyl-1-octyl-5-phenyl-1H-pyrrol-3-yl)ethano ne (4d): Yellow oil; ${ }^{1} \mathrm{H}$ NMR $\left(300 \mathrm{MHz}, \mathrm{CDCl}_{3}\right): \delta$ (ppm) $7.53-7.21(\mathrm{~m}, 5 \mathrm{H}), 6.65(\mathrm{~s}, 1 \mathrm{H}), 3.77(\mathrm{t}, \mathrm{J}=7 \mathrm{~Hz}$, 2H), 2.47 (s, 3H), $2.38(\mathrm{~s}, 3 \mathrm{H}), 1.79$ - 1.67 (m, 4H), 1.02 $0.95(\mathrm{~m}, 8 \mathrm{H}), 0.80(\mathrm{t}, \mathrm{J}=7 \mathrm{~Hz}, 3 \mathrm{H}) ; \mathrm{MS}(\mathrm{ESI}): \mathrm{m} / \mathrm{z}=312$ $[\mathrm{M}+1]^{+}$; IR (KBr): 2944, 1695, 1450, 1600, $1223 \mathrm{~cm}^{-1}$. 


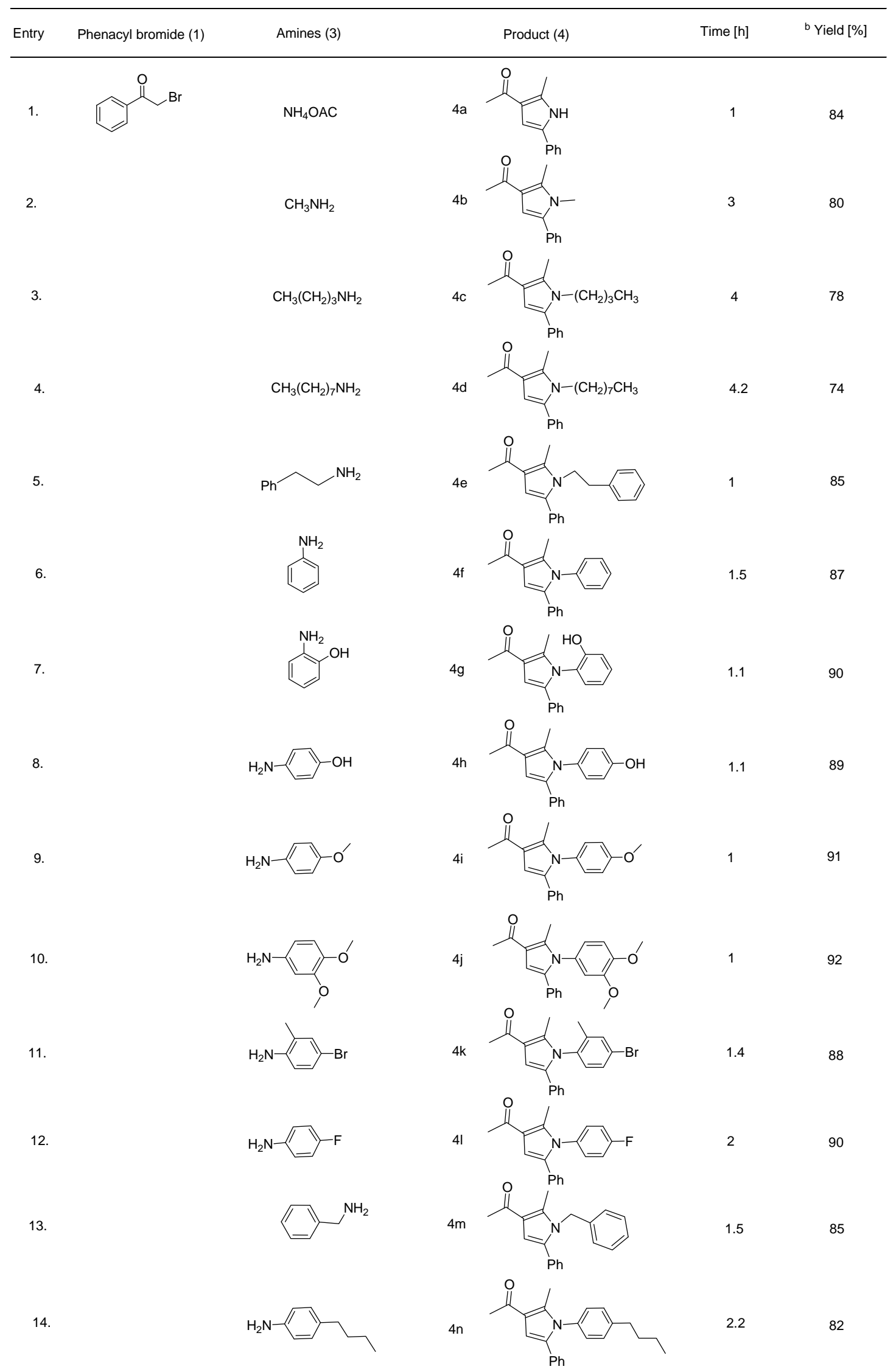


15.

16.

17.

18.

19<smiles>O=C(CBr)c1ccc(-c2ccccc2)cc1</smiles>

20

22

23.

24.

25.
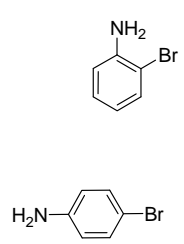<smiles>CCc1cccc(CC)c1N</smiles><smiles>Nc1ccc(Cl)c(Cl)c1</smiles><smiles>Nc1ccc(Cl)c(Cl)c1</smiles><smiles>Nc1ccc(Cl)c(Cl)c1</smiles>

$\mathrm{CH}_{3} \mathrm{NH}_{2}$<smiles>NCCc1ccccc1</smiles><smiles>Cc1cc(Br)ccc1N</smiles><smiles>Nc1ccc(F)cc1</smiles><smiles>Nc1ccccc1O</smiles>

40<smiles>CC(=O)c1cc(-c2ccccc2)n(-c2ccccc2Br)c1C</smiles>

$4 p$<smiles>CC(=O)c1cc(-c2ccccc2)n(-c2ccc(Br)cc2)c1-c1ccccc1</smiles>

$4 q$<smiles>CCc1cccc(CC)c1-n1c(-c2ccccc2)cc(C(C)=O)c1C</smiles>

$4 r$<smiles>CC(=O)c1cc(-c2ccccc2)n(-c2ccc(Cl)c(Cl)c2)c1C</smiles>

$4 s$<smiles>CC(=O)c1cc(-c2ccccc2)n(-c2ccc(Cl)c(Cl)c2)c1C</smiles><smiles>CC(=O)c1cc(-c2ccc(Br)cc2)n(-c2ccc(Cl)c(Cl)c2)c1C</smiles>

$4 u$<smiles>CC(=O)c1cc(-c2ccc(Br)cc2)n(C)c1C</smiles>

$4 \mathrm{v}$<smiles>CC(=O)c1cc(-c2ccc(Br)cc2)n(CCc2ccccc2)c1C</smiles>

$4 w$<smiles>CC(=O)c1cc(-c2ccc(Br)cc2)n(-c2ccc(Br)cc2C)c1C</smiles>

$4 x$<smiles>CC(=O)c1cc(-c2ccc(Br)cc2)n(-c2ccc(F)cc2)c1C</smiles>

$4 y$
1.7

88

Figure 1. Synthesis ${ }^{\mathrm{a}}$ of substituted pyrroles. ${ }^{\mathrm{a}}$ Reaction condition: pantane-2,3-dione (1 mmol), amine (1 mmol), phenacyl bromide $(1 \mathrm{mmol})$ and DABCO $(10 \mathrm{~mol} \%)$ in $5 \mathrm{ml}$ water at $60^{\circ} \mathrm{C}$; ${ }^{\mathrm{b}}$ Yield of isolated products. 


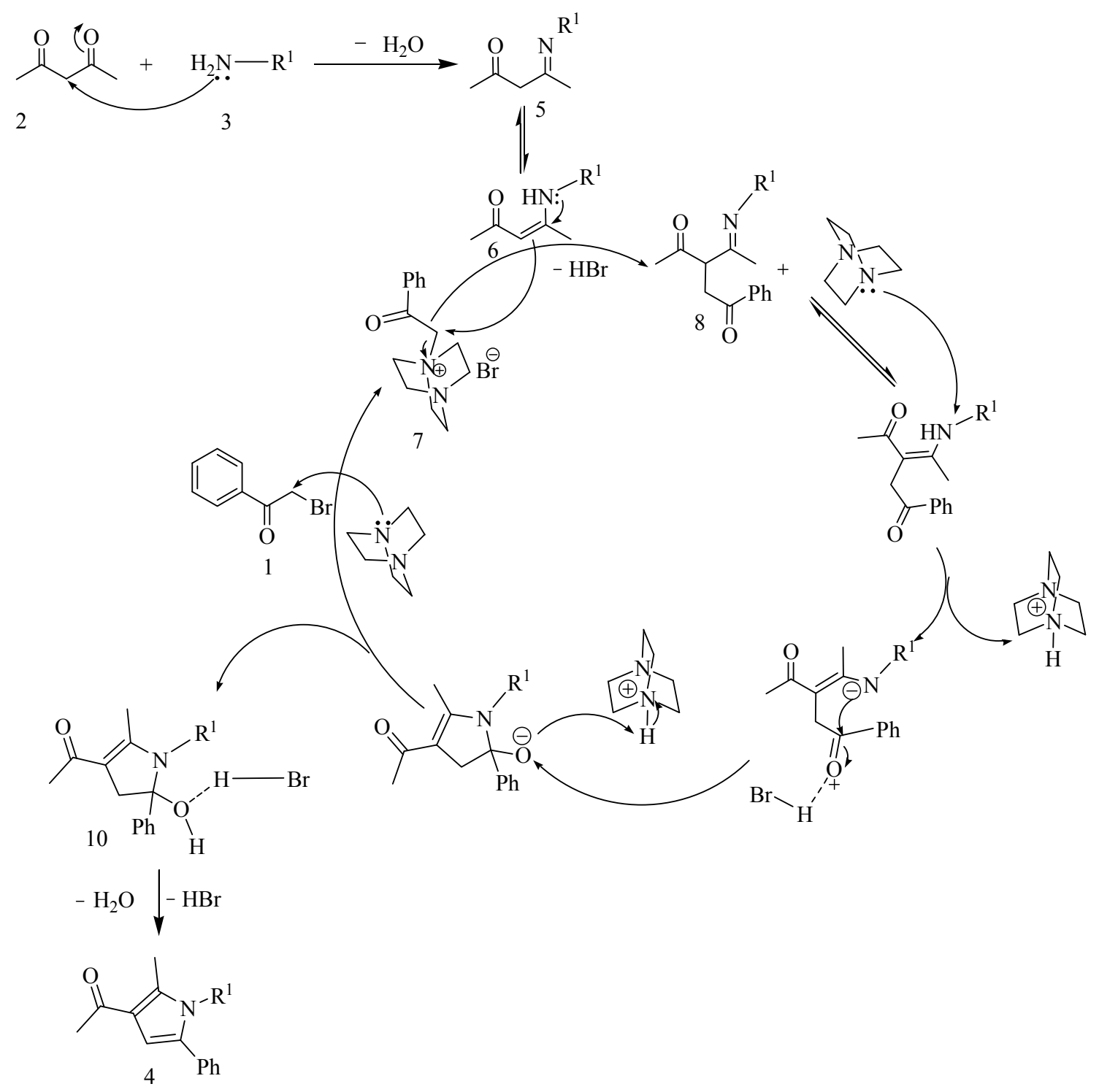

Scheme 2. Plausible mechanism.

1-(2-methyl-1-phenethyl-5-phenyl-1H-pyrrol-3-yl) ethanone (4e): Yellow oil; ${ }^{1} \mathrm{H}$ NMR $\left(300 \mathrm{MHz}, \mathrm{CDCl}_{3}\right)$ : $\delta(\mathrm{ppm}) 7.40-7.20(\mathrm{~m}, 10 \mathrm{H}), 6.36(\mathrm{~s}, 1 \mathrm{H}), 4.04(\mathrm{t}, \mathrm{J}=7$ $\mathrm{Hz}, 2 \mathrm{H}), 3.00$ (t, J = 7 Hz, 2H), 2.38 (s, 3H), $2.26(\mathrm{~s}, 3 \mathrm{H})$; MS (ESI): $\mathrm{m} / \mathrm{z}=304[\mathrm{M}+1]^{+}$; IR (KBr): 3060, 3027, $1649,1501,1415 \mathrm{~cm}^{-1}$.

1-(1-(2-hydroxyphenyl)-2-methyl-5-phenyl-1H-pyrr ol-3-yl)ethanone (4g): Yellow oil; ${ }^{1} \mathrm{H}$ NMR $(300 \mathrm{MHz}$, $\left.\mathrm{CDCl}_{3}\right): \delta(\mathrm{ppm}) 7.20-6.90(\mathrm{~m}, 9 \mathrm{H}), 6.57(\mathrm{~s}, 1 \mathrm{H}), 6.05$ (s, 1H), 2.30 (s, 3H), 2.06 (s, 3H); MS (ESI): $\mathrm{m} / \mathrm{z}=314$ $[\mathrm{M}+\mathrm{Na}]^{+}$; IR (KBr): 3341, 2924, 1708, 1599, $1495 \mathrm{~cm}^{-1}$.

1-(1-(4-hydroxyphenyl)-2-methyl-5-phenyl-1H-pyrr ol-3-yl)ethanone (4h): Yellow oil; ${ }^{1} \mathrm{H}$ NMR (300 MHz, $\left.\mathrm{CDCl}_{3}\right): \delta(\mathrm{ppm}) 9.86(\mathrm{~s}, 1 \mathrm{H}), 7.51-7.12(\mathrm{~m}, 9 \mathrm{H}), 6.57$ (s, 1H), 2.27 (s, 3H), 2.06 (s, 3H); MS (ESI): $\mathrm{m} / \mathrm{z}=314$ $[\mathrm{M}+\mathrm{Na}]^{+}$; IR (KBr): 3331, 2994, 1665, 1519, $1220 \mathrm{~cm}^{-1}$. 1-(1-(4-bromo-2-methylphenyl)-2-methyl-5-phenyl1H-pyrrol-3-yl)ethanone (4k): Yellow oil; ${ }^{1} \mathrm{H}$ NMR (300 $\left.\mathrm{MHz}, \mathrm{CDCl}_{3}\right): \delta(\mathrm{ppm}) 7.49-7.13(\mathrm{~m}, 8 \mathrm{H}), 6.63(\mathrm{~s}, 1 \mathrm{H})$, $2.45(\mathrm{~s}, 3 \mathrm{H}), 2.42$ (s, 3H), 2.35 (s, 3H); MS (ESI): $\mathrm{m} / \mathrm{z}=$ 368 [M] $]^{+}$IR (KBr): 3050, 3012, 1677, 1612, 1405, 1220, $753,697 \mathrm{~cm}^{-1}$.

1-(1-(2-bromophenyl)-2-methyl-5-phenyl-1H-pyrrol -3-yl)ethanone (4o): Yellow oil; ${ }^{1} \mathrm{H}$ NMR $(300 \mathrm{MHz}$, $\left.\mathrm{CDCl}_{3}\right): \delta(\mathrm{ppm}) 8.06-7.17(\mathrm{~m}, 9 \mathrm{H}), 6.62(\mathrm{~s}, 1 \mathrm{H}), 2.56$ (s, 3H), 2.39 (s, 3H); MS (ESI): m/z = $354[\mathrm{M}+1]^{+}$; IR (KBr): 3012, 1677, 1612, 1405, 1220, 753, $697 \mathrm{~cm}^{-1}$.

1-(1-(4-bromophenyl)-2-methyl-5-phenyl-1H-pyrrol -3-yl)ethanone (4p): Yellow oil; ${ }^{1} \mathrm{H}$ NMR $(300 \mathrm{MHz}$, $\left.\mathrm{CDCl}_{3}\right): \delta(\mathrm{ppm}) 8.06-7.72(\mathrm{~m}, 9 \mathrm{H}), 6.67(\mathrm{~s}, 1 \mathrm{H}), 2.38$ (s, 3H), 2.06 (s, 3H); MS (ESI): m/z = $354[\mathrm{M}+1]^{+}$; IR (KBr): 2992, 1654, 1595, 1450, 1313, 790, $715 \mathrm{~cm}^{-1}$. 
1-(1-(3,4-dichlorophenyl)-2-methyl-5-phenyl-1H-py rrol-3-yl)ethanone (4r): Yellow oil; ${ }^{1} \mathrm{H}$ NMR $(300 \mathrm{MHz}$, $\left.\mathrm{CDCl}_{3}\right): \delta(\mathrm{ppm}) 7.45-7.13(\mathrm{~m}, 8 \mathrm{H}), 6.64(\mathrm{~s}, 1 \mathrm{H}), 2.45$ (s, 3H), 2.43 (s, 3H); MS (ESI): $\mathrm{m} / \mathrm{z}=344[\mathrm{M}]^{+}$; IR (KBr): $3029,1715,1545,1415,789,720 \mathrm{~cm}^{-1}$.

1-(5-(biphenyl-4-yl)-1-(3,4-dichlorophenyl)-2-methy l-1H-pyrrol-3-yl)ethanone (4s): Yellow oil; ${ }^{1} \mathrm{H}$ NMR $\left(300 \mathrm{MHz}, \mathrm{CDCl}_{3}\right): \delta(\mathrm{ppm}) 7.53-7.15(\mathrm{~m}, 12 \mathrm{H}), 6.70$ (s, $1 \mathrm{H}), 2.48$ (s, 3H), 2.45 (s, 3H); MS (ESI): $\mathrm{m} / \mathrm{z}=420$ [M] $]^{+}$IR (KBr): 2964, 1659, 1514, $770 \mathrm{~cm}^{-1}$.

1-(5-(4-bromophenyl)-1-(3,4-dichlorophenyl)-2-met hyl-1H-pyrrol-3-yl)ethanone (4t): Yellow oil; ${ }^{1} \mathrm{H}$ NMR $\left(300 \mathrm{MHz}, \mathrm{CDCl}_{3}\right): \delta(\mathrm{ppm}) 7.56-7.14(\mathrm{~m}, 7 \mathrm{H}), 6.65(\mathrm{~s}$, $1 \mathrm{H}), 2.45$ (s, 3H), 2.42 (s, 3H); MS (ESI): $\mathrm{m} / \mathrm{z}=424[\mathrm{M}$ $+1]^{+}$; IR (KBr): 3112, 1689, 1495, 812, 799, $712 \mathrm{~cm}^{-1}$.

1-(5-(4-bromophenyl)-1,2-dimethyl-1H-pyrrol-3-yl) ethanone (4u): Yellow oil; ${ }^{1} \mathrm{H}$ NMR $\left(300 \mathrm{MHz}, \mathrm{CDCl}_{3}\right)$ : $\delta(\mathrm{ppm}) 7.53-7.13(\mathrm{~m}, 4 \mathrm{H}), 6.44(\mathrm{~s}, 1 \mathrm{H}), 2.38(\mathrm{~s}, 3 \mathrm{H})$, 2.32 (s, 3H), 2.30 (s, 3H); MS (ESI): $\mathrm{m} / \mathrm{z}=293[\mathrm{M}+1]^{+}$; IR (KBr): 2924, 1654, 1595, 1405, $1223 \mathrm{~cm}^{-1}$.

1-(5-(4-bromophenyl)-2-methyl-1-phenethyl-1H-py rrol-3-yl)ethanone (4v): Yellow oil; ${ }^{1} \mathrm{H}$ NMR (300 MHz, $\left.\mathrm{CDCl}_{3}\right): \delta(\mathrm{ppm}) 7.53-7.13(\mathrm{~m}, 9 \mathrm{H}), 6.44(\mathrm{~s}, 1 \mathrm{H}), 2.99(\mathrm{t}$, $\mathrm{J}=7 \mathrm{~Hz}, 2 \mathrm{H}), 2.86(\mathrm{t}, \mathrm{J}=7 \mathrm{~Hz}, 2 \mathrm{H}), 2.54(\mathrm{~s}, 3 \mathrm{H}) 2.38(\mathrm{~s}$, $3 \mathrm{H}) ; \mathrm{MS}(\mathrm{ESI}): \mathrm{m} / \mathrm{z}=382[\mathrm{M}]^{+}$; IR (KBr): 2920, 1654, $1595,1415,1203,717 \mathrm{~cm}^{-1}$.

1-(1-(4-bromo-2-methylphenyl)-5-(4-bromophenyl)2-methyl-1H-pyrrol-3-yl)ethanone (4w): Yellow oil; ${ }^{1} \mathrm{H}$ NMR $\left(300 \mathrm{MHz}, \mathrm{CDCl}_{3}\right): \delta(\mathrm{ppm}) 7.48-7.12(\mathrm{~m}, 7 \mathrm{H})$, $6.71(\mathrm{~s}, 1 \mathrm{H}), 2.53(\mathrm{~s}, 3 \mathrm{H}), 2.43(\mathrm{~s}, 3 \mathrm{H}), 2.37$ (s, 3H); MS (ESI): $\mathrm{m} / \mathrm{z}=448[\mathrm{M}+1]^{+}$; IR (KBr): 2924, 1695, 1551, $1400,1223,727 \mathrm{~cm}^{-1}$.

1-(5-(4-bromophenyl)-1-(4-fluorophenyl)-2-methyl1H-pyrrol-3-yl)ethanone (4x): Yellow oil; ${ }^{1} \mathrm{H}$ NMR (300 $\left.\mathrm{MHz}, \mathrm{CDCl}_{3}\right): \delta(\mathrm{ppm}) 7.43-7.11(\mathrm{~m}, 8 \mathrm{H}), 6.78(\mathrm{~s}, 1 \mathrm{H})$, 2.52 (s, 3H), 2.37 (s, 3H); MS (ESI): $\mathrm{m} / \mathrm{z}=373[\mathrm{M}+1]^{+}$; IR (KBr): 3029, 1716, 1455, 1239, 745, $723 \mathrm{~cm}^{-1}$.

1-(5-(4-bromophenyl)-1-(2-hydroxyphenyl)-2-meth yl-1H-pyrrol-3-yl)ethanone (4y): Yellow oil; ${ }^{1} \mathrm{H}$ NMR $\left(300 \mathrm{MHz}, \mathrm{CDCl}_{3}\right): \delta(\mathrm{ppm}) 7.42-7.12(\mathrm{~m}, 8 \mathrm{H}), 6.85(\mathrm{~s}$, $1 \mathrm{H}), 6.65(\mathrm{~s}, 1 \mathrm{H}), 2.58(\mathrm{~s}, 3 \mathrm{H}), 2.37(\mathrm{~s}, 3 \mathrm{H})$; MS (ESI): $\mathrm{m} / \mathrm{z}=373[\mathrm{M}+1]^{+} ; \mathrm{IR}(\mathrm{KBr}): 3411,3024,1654,1595$, $1405,789 \mathrm{~cm}^{-1}$.

\section{Conclusion}

In summary, we have demonstrated a simple, general practical approach for the synthesis of substituted pyrroles. The method is applicable for a variety of alkyl, aryl and benzylic amines as well as for substituted phenacyl halides which can provide easy access to required substituted pyrroles. The method avoids the metal containing toxic waste and thus makes the procedure more advantage. Moreover, the use of aqueous medium makes the procedure more eco-friendly.

\section{Acknowledgements}

V. M. B., B. C. K. R., G. S. K. and P. B. T. thanks CSIRUGC for the award of a fellowship and to Dr. J. S. Yadav, Director IICT, for his support and encouragement.

\section{REFERENCES}

[1] R. A. Jones and G. P. Bean, "The Chemistry of Pyrroles," Academic Press, London, 1977.

[2] R. J. Sundberg, "Comprehensive Heterocyclic Chemistry," Pergamon Press, Oxford, 1984, pp. 313-379. doi:10.1016/B978-008096519-2.00056-4

[3] D. L. Boger, C. W. Boyce, M. A. Labroli, C. A. Sehon and Q. Jin, "Total Synthesis of Ningalin A, Lamellarin O, Lukianol A, and Permethyl Storniamide A Utilizing Heterocyclic Azadiene Diels-Alder Reactions," Journal of the American Chemical Society, Vol. 121, No. 1, 1999, pp. 54-62. doi:10.1021/ja982078+

[4] L. F. Tietze and G. Nordmann, "Synthesis of a Linear Oligomeric Styrylpyrrole Using Multiple Heck and Wittig Reactions," Synlett, No. 3, 2001, pp. 337-340.

[5] L. Groenendaal, E. W. Meijer and J. A. J. M. Vekemans, "Electronic Materials: The Oligomer Approach," K. M. Allen and G. Wegner, Eds., Wiley-VCH, Weinheim, 1997.

[6] J. J. Kulagowski, H. B. Broughton, N. R. Curtis, I. M. Mawer, M. P. Ridgill, R. Baker, F. Emms, S. B. FreedMan, R. Marwood, S. Patel, C. I. Ragan and P. D. Leeson, "3[4-(4-Chlorophenyl)-piperazin-1-yl]-1H-pyrrolo[2,3-b] Pyridine: An Antagonist with High Affinity and Selectivity for the Human Dopamine D-4 Receptor," Journal of Medicinal Chemistry, Vol. 39, No. 10, 1996, pp. 19411942. doi: $10.1021 / \mathrm{jm} 9600712$

[7] J. R. Henry, K. C. Rupert, J. H. Dodd, I. J. Turchi, S. A. Wadsworth, D. E. Cavender, B. Fahmy, G. C. Olini, J. E. Davis, J. L. P. Genesy, P. H. Schafer and J. J. Siekierka, "6-Amino-2-(4-fluorophen-yl)-4-metho-xy-3-(4-pyridyl)1H-pyrrolo[2,3-b]pyridine (RNJ 68354): A Potent and Selective P-38 Kinase Inhibitor," Journal of Medicinal Chemistry, Vol. 41, No. 22, 1998, pp. 4196-4198. doi: $10.1021 / \mathrm{jm} 980497 \mathrm{~b}$

[8] I. A. Schepetkin, A. I. Khlebnikov and M. T. Quinn, "NBenzoylpyrazoles Are Novel Small-Molecule Inhibitors of Human Neutrophil Elastase," Journal of Medicinal Chemistry, Vol. 50, No. 20, 2007, pp. 4928-4938. doi:10.1021/jm070600+

[9] A. Miszke, H. Foks, A. Kedazia, E. Kwapisz and Z. Zwolska, "The Synthesis and Microbiological Activity of 2-Mercapto-4-(pyrrolidin-1-yl)pyridine Derivatives," Heterocycles, Vol. 75, No. 9, 2008, pp. 2251-2261.

[10] V. K. Alexander, A. W. Sromek and V. Gevorgyan, "A Novel Cu-Assisted Cycloisomerization of Alkynyl Imines: Efficient Synthesis of Pyrroles and Pyrrole-Containing Heterocycles," Journal of the American Chemical Society, Vol. 123, No. 9, 2001, pp. 2074-2075. doi: $10.1021 / \mathrm{ja} 0058684$

[11] J. T. Binder and S. F. Kirsch, "Synthesis of Highly Substituted Pyrroles via a Multimetal-Catalyzed Rearrangement-Condensation-Cyclization Domino Approach," Or- 
ganic Letters, Vol. 8, No. 10, 2001, pp. 2151-2153.

[12] H. Shiraishi, T. Nishitani, S. Sakaguchi and Y. Ishii, "Preparation of Substituted Alkylpyrroles via SamariumCatalyzed Three-Component Coupling Reaction of Aldehydes, Amines, and Nitroalkanes," The Journal of Organic Chemistry, Vol. 63, No. 18, 1998, pp. 6234-6238. doi:10.1021/jo980435t

[13] S. N. Murthy, B. Madhav, A. V. Kumar, K. R. Rao and Y. V. D. Nageswar, "Multicomponent Approach Towards the Synthesis of Substituted Pyrroles under Supramolecular Catalysis Using b-Cyclodextrin as a Catalyst in Water Under Neutral Conditions," Helvetica Chimica Acta, Vol. 92, 2009, pp. 2118-2124. doi:10.1002/hlca.200900098

[14] P. T. Anastas and J. C. Warner, "Green Chemistry: Theory and Practice," Oxford University Press, New York, 1998, p. 30.

[15] C. J. Li, "Organic Reactions in Aqueous Media with a Focus on Carbon-Carbon Bond Formations: A Decade Update," Chemical Review, Vol. 105, No. 8, 2005, pp. 30953166. doi: $10.1021 / \mathrm{cr} 030009 \mathrm{u}$

[16] C. Zorn, F. Gnad, S. Salmen, T. Herpin and O. Reiser, "Deprotection of N-Alloc amines by $\mathrm{Pd}(0) / \mathrm{DABCO}$-An Efficient Method for in Situ Peptide Coupling of Labile Amino Acids," Tetrahedron Letters, Vol. 42, No. 40, 2001, pp. 7049-7053. doi:10.1016/S0040-4039(01)01453-8

[17] P. R. Krishna, E. R. Sekhar and V. Kannan, "The Use of Acetylenic Aldehydes in Baylis-Hillman Reactions: Synthesis of Versatile Allyl Propargyl Alcohols," Tetrahedron Letters, Vol. 44, No. 27, 2003, pp. 4973-4975.

[18] A. Kumar and S. S. Pawar, "Salt Effects on the BaylisHillman Reaction," Tetrahedron, Vol. 59, No. 27, 2003, pp. 5019-5026. doi:10.1016/S0040-4020(03)00760-9

[19] L. Cecchi, F. D. Sarlo and F. Machetti, "1,4-Diazabicyclo [2.2.2]octane (DABCO) as an Efficient Reagent for the Synthesis of Isoxazole Derivatives from Primary Nitro Compounds and Dipolarophiles: The Role of the Base," European Journal of the Organic Chemistry, Vol. 2006, No. 21, 2006, pp. 4852-4860. doi:10.1002/ejoc. 200600475

[20] X. Bu, H. Jing, L. Wang, T. Chang, L. Jin and Y. Liang, "Organic Base Catalyzed O-Alkylation of Phenols under Solvent-Free Condition," Journal of Molecular Catalysis A: Chemical, Vol. 259, No. 1-2, 2006, pp. 121-124. doi:10.1016/j.molcata.2006.06.009

[21] T. Sharafi and M. M. Heravi, "DABCO, A Reagent for Deprotection of Benzylic Trimethylsilyl Ethers under Microwave Irradiation in a Solventless System," Phosphorus, Sulfur Silicon, Vol. 179, No. 12, 2004, pp. 2437-2440.

\section{doi:10.1080/10426500490485327}

[22] R. S. Varma, R. K. Saini and H. M. Meshram, "Selective Oxidation of Sulfides to Sulfoxides and Sulfones by Microwave Thermolysis on Wet Silica-Supported Sodium Periodate," Tetrahedron Letters, Vol. 38, No. 37, 1997, pp. 6525-6528. doi:10.1016/S0040-4039(97)01520-7

[23] H. M. Meshram, D. Srinivas and J. S. Yadav, "A General Synthesis of Isothiocyanates from Dithiocarbamates Using Claycop," Tetrahedron Letters, Vol. 38, No. 50, 1997, pp. 8743-8744. doi:10.1016/S0040-4039(97)10158-7

[24] H. M. Meshram, G. S. Reddy and J. S. Yadav, "Clay Supported Ammonium Nitrate 'Clayan': A Mild and EcoFriendly Reagent for Dethioacetalization,” Tetrahedron Letters, Vol. 38, No. 51, 1997, pp. 8891-8894. doi:10.1016/S0040-4039(97)10349-5

[25] H. M. Meshram, G. S. Reddy, M. M. Reddy and J. S. Yadav, "Zinc Mediated Facile Amide Formation: Application to Alkyl, Aryl, Heterocycle, Carbohydrate and Amino Acids," Tetrahedron Letters, Vol. 39, No. 23, 1998, pp. 4103-4106. doi:10.1016/S0040-4039(98)00666-2

[26] H. M. Meshram, B. C. Reddy and P. R. Goud, "DABCO Promoted an Efficient Synthesis of Benzofurans," Synthetic Communications, Vol. 39, 2009, pp. 2297-2303. doi: 10.1080/00397910802654740

[27] H. M. Meshram, G. S. Kumar, P. Ramesh and B. C. Reddy, "One-Pot Synthesis of Quinoxaline-2-Carboxylate Derivatives Using Ionic Liquid as Reusable Reaction Media," Tetrahedron Letters, Vol. 51, No. 33, 2010, pp. 4313-4316. doi:10.1016/j.tetlet.2010.05.099

[28] H. M. Meshram, P. Ramesh, G. S. Kumar and B. C. Reddy, "A Mild and Convenient Synthesis of Quinoxalines via Cyclization-Oxidation Process Using DABCO as Catalyst," Tetrahedron Letters, Vol. 51, No. 19, 2010, pp. 2580-2585. doi:10.1016/j.tetlet.2010.01.107

[29] R. Dalpozzo, A. D. Nino, M. Nardi, B. Russo and A. Procopio, "Erbium(III) Triflate: A Valuable Catalyst for the Synthesis of Aldimines, Ketimines, and Enaminones," Synthesis, No. 7, 2006, pp. 1127-1132. doi:10.1055/s-2006-926378

[30] V. Sridharan, C. Avendano and J. C. Menendez, "General, Mild and Efficient Synthesis of $\beta$-Enaminones Catalyzed by Ceric Ammonium Nitrate," Synlett, No. 6, 2007, pp. 881-884.

[31] M. Fan, L. Guo, X. Liu, W. Liu and Y. Liang, "A Mild, Convenient and Efficient Single-Step Method for the Synthesis of Polysubstituted Furans via Ammonium Ylide Routes," Synthesis, No. 3, 2005, pp. 391-396. 